\title{
Deciding when to intrude on a neighbour: quantifying behavioural mechanisms for temporary territory expansion
}

\author{
Jonathan R. Potts ${ }^{1}$ (1) . William F. Fagan ${ }^{2}$. Guilherme Mourão ${ }^{3}$
}

Received: 29 March 2018 / Accepted: 4 October 2018 / Published online: 25 October 2018

(C) The Author(s) 2018

\begin{abstract}
Flexible territorial structures are common to a variety of animal populations. When resources are abundant, animals can maintain relatively fixed territory boundaries. However, if resources decline, animals may have to intrude temporarily into a neighbour's territory to secure enough food for survival. Although such intrusions may be necessary, they take time away from foraging and can lead to costly conflicts, resulting in a behavioural trade-off. Here, we examine this trade-off using a spatially explicit, energy-based movement model inspired by observations of giant otters. We uncover conditions under which temporary neighbour intrusions are beneficial. We show that, under certain circumstances, this benefit is sufficient for allowing territorial groups to survive in perpetuity, when otherwise they would be forced to disperse or die. Our model also reveals plausible mechanisms behind a variety of observed phenomena, including the emergence of intermediate-sized territorial groups, territorial fission/fusion dynamics, and the employment of multiple methods for advertising territories (e.g. vocal and olfactory). Although we focus our modelling on giant otters, the behavioural mechanisms it describes are quite general, having been observed across a wide range of taxa, including birds, fish and mammals. Our model therefore serves as a general theoretical test-bed for understanding temporary territory expansion.
\end{abstract}

Keywords Aggressive behaviour · Giant otters · Movement ecology $\cdot$ Scent-marking $\cdot$ Spatial ecology $\cdot$ Territoriality

\section{Introduction}

Many species in the animal kingdom exhibit territoriality, whereby sub-groups of a population secure areas of space for exclusive use for themselves, whilst actively excluding others (Burt 1943; Adams 2001; Potts and Lewis 2014). The act of conspecific exclusion leads to an emergent 'agreement' between adjacent territory owners which may persist across a season or longer, causing stable spatial

Electronic supplementary material The online version of this article (https://doi.org/10.1007/s12080-018-0396-x) contains supplementary material, which is available to authorized users.

Jonathan R. Potts

j.potts@sheffield.ac.uk

1 School of Mathematics and Statistics, University of Sheffield, Hicks Building, Hounsfield Road, Sheffield, UK, S3 7RH, USA

2 Department of Biology, University of Maryland College Park, College Park, MD 20742, USA

3 Embrapa Pantanal, Rua 21 de setembro 1880, Corumbá, MS, 79320-900, Brazil segregation patterns to form (Lewis and Moorcroft 2006). A basic life-history requirement for stable territories to emerge is that they are sufficiently large to sustain the animal's foraging needs (Mitchell and Powell 2004). Therefore, as long as the resource distribution remains roughly constant over time, there is no need for animals to break this 'agreement' and intrude into others' territories, at least from a foraging perspective.

However, as seasons shift, the resources within a territory may change (Winemiller and Jepsen 1998; Zhou and Fagan 2017). This can leave individuals unable to sustain themselves by foraging within their territories (Leuchtenberger et al. 2015). To cope with this, species employ a variety of different strategies. These include extraterritorial forays (Evans et al. 2008; Patterson and Messier 2001), attempts to take-over a neighbouring territory (Wirtz 1981; Piper et al. 2000), or extensions of an existing territory to access greater resources (Roper et al. 1986; Leuchtenberger et al. 2015).

In this paper, we examine the costs and benefits of pursuing territorial extensions. In particular, we consider scenarios in which long-term marking of territorial boundaries is temporarily supplemented by a second, more transient mode 
of boundary marking with different costs. We base our analysis on observations of a specific population of giant otters (Pteronura brasiliensis), which live in rivers within the Pantanal, a tropical wetland area of South America. Giant otters are apex predators that can reach up to $1.8 \mathrm{~m}$ long, and adults can weigh between 20 and $32 \mathrm{~kg}$. They feed mainly on fish (Duplaix 1980) and each otter can consume up to 4 $\mathrm{kg}$ of food per day (Carter and Rosas 1997).

Giant otters live in social groups of around 2 to 20 individuals, formed usually, but not always, by an unrelated reproductive pair and kin non-reproductive helpers. Each group maintains a territory along a section of river. Territories are demarcated by latrines, or other type of scent marks of a group, at either end (Leuchtenberger et al. 2015). Typically, latrines or scent marks of one group will abut those of another, leading to a contiguous sequence of territories along the river (Fig. 1). Such contiguous territories were observed, for example, in the high-water season of 2010 (Leuchtenberger et al. 2015).

In 'times of plenty', when each non-overlapping territory is sufficient to sustain the group therein, these territories remain contiguous with fixed borders. However, in 'lean times', a competition emerges, as one territorial group seeks to push its borders back, possibly causing the adjacent group to respond in kind. During these times, otters make excursions beyond the neighbouring group's latrines, often making loud vocalisations to announce their presence. These excursions give rise to fluctuations in territorial structure, suggesting otters are able to secure extra-territorial area for a period of time through vocal cues, a tactic employed by a variety of territorial species (Adams 2001).

The resulting overlap in space use causes neighbouring groups to encounter one another. For example, overlapping territories have been observed in the low-water season of 2009 and high-water season of 2011 (Leuchtenberger et al. 2015). The aggressive nature of these interactions results in energetic loss (Ribas and Mourão 2004; Leuchtenberger and Mourão 2009), which trades-off against the energetic gains from greater access to forage. We examine the conditions under which temporary extensions of territories, caused by vocalisations beyond the latrines, leads to a beneficial outcome for giant otters. We also quantify the extent of that benefit in some simple examples.

We use a spatially explicit modelling approach to assess the trade-off between these costs and benefits. The aim is to understand the situations in which the use of temporary territorial extensions will be beneficial to giant otter survival, and when they would be unnecessary or detrimental. The model explicitly incorporates movement and interaction of individuals, building on ideas from Giuggioli et al. (2011) and Potts et al. (2012). Foraging is modelled as a persistent random walk within territorial borders which can shift over time (Giuggioli et al. 2012; Potts et al. 2012). However, unlike those previous papers, here, the movement of the borders is not random but determined by whether or not the individuals have an energetic need to shift the borders, and so obtain access to a greater quantity of forage.

In particular, we explore three key questions, namely (i) how many individuals can a given area sustain without the need to move territory borders, (ii) to what extent can temporary territorial extensions improve the existence time of a group in the area, and (iii) in a competition scenario, where two adjacent groups need to extend their territories temporarily to survive, can these extensions increase the coexistence time between groups (as opposed to the survival time of a single group out-competing the other)? The spatially explicit nature of our modelling approach enables us to link the spatial extent of territorial extensions with the ability for animal populations to extend their longevity, which is not possible with non-spatial models.

Although we focus on a population of giant otters, we intentionally construct a model that should be generally applicable to a range of territorial animals. To apply our model to a given population, the key pre-requisites are that each territorial unit in the population (a) maintains clearly demarcated borders and (b) occasionally extends its territory temporarily (e.g. due to seasonal fluctuations in resource availability). Such features have been observed in many species, including canids (Messier 1985; Patterson and Messier 2001), birds (Naguib et al. 2001), fish (Bartels 1984; Craig 1996), and badgers (Evans et al. 1989; Delahay et al. 2000). Therefore the model and results presented here have potential for wide application.

\section{The model}

Our model considers giant otters living in a river that is relatively narrow compared to its length. Therefore the model is constructed on a one-dimensional line segment. This segment is partitioned into territories, each one containing a single otter group. At each end of each territory is a set of latrines which the otters mark periodically to demarcate the border (see Fig. 1).

Occasionally, otters may make excursions beyond their latrines, using vocalisations (Calls) to advertise their presence to the neighbouring group. This has the effect of temporarily extending their territory as far as a Calling Point, denoted $C_{i}$ for group $i$. This gives the group access to more resources for a period of time. During this time, we assume the adjacent group takes on a subordinate role, refusing to pass the calling point and being temporarily left with a smaller territory (Leuchtenberger et al. 2015). For the purposes of our analysis, it suffices to model the 


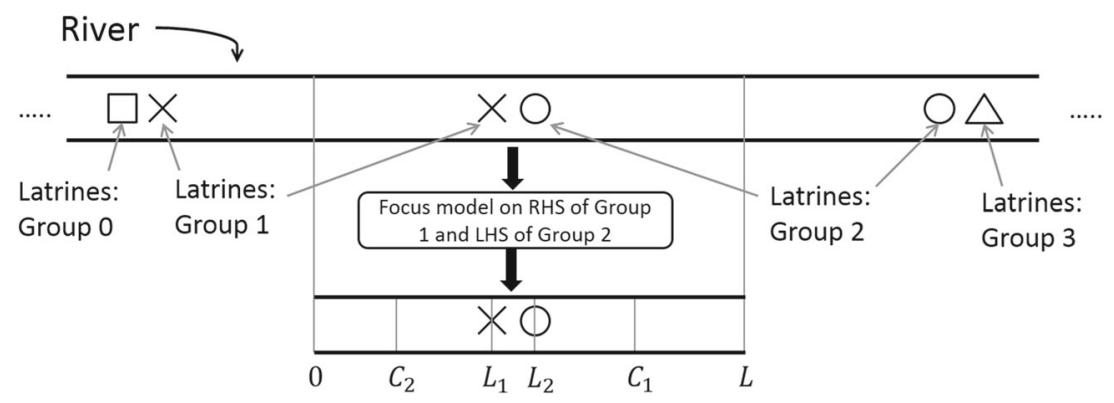

Fig. 1 Pictorial representation of the model. The river is modelled as a line, which contains contiguous territories of different otter groups. Each territory is demarcated by latrines at either end. The top picture shows an example containing latrines from four otter territories. For the purposes of this paper, it suffices to focus on the right-hand side of territory 1 and the left-hand side of territory 2 , shown in the bottom picture. The right-most latrine of group 1 is at position $L_{1}$ and the leftmost latrine of group 2 at position $L_{2}$. Although $L_{1}$ and $L_{2}$ are shown as distinct points, territories will typically abut so, in our analysis, we always have $L_{1}=L_{2}$. Group 1 (resp. group 2) may occasionally seek to extend its territory through making a vocal cue at $C_{1}$ (resp. $C_{2}$ )

they tend to swim in the same direction (upstream or downstream) for around an hour at a time (GM, pers. obs.).

When Foraging, the group performs a bounded persistent random walk with reflecting boundary conditions, and fixed persistence time $\tau$ (note: this is a discrete-time simplification of the general definition of a persistent random walk, where the persistence time can vary about a characteristic mean time, e.g. Masoliver et al. 1989). This means that the direction of movement is chosen at random each time-step (with equal probability of moving right or left), except when the edge of the territory is reached, at which point the movement is reflected back from where the group came. Notice that $\tau$ is importantly related to the biology of the animal, since there will be a natural persistence in movement due to effort it takes to change direction (Wilson et al. 2013). Moreover, since persistence in movement can affect territorial dynamics (Giuggioli et al. 2012), we would expect the choice of $\tau$ to have consequences for the model output, so cannot be treated as an arbitrary parameter.

When Scenting, group $i$ (for $i=1$ or 2 ) moves directly towards the chosen latrine. When group $i$ reaches the end of the latrine zone, at point $L_{i}$ (see Fig. 1), the latrines are assumed to be 'marked' and the group reverts to Foraging. Likewise, when Calling, group $i$ moves directly towards the chosen calling point, $C_{i}$. This group then reverts to Foraging once $C_{i}$ has been reached. At the point when $C_{i}$ is reached, we say that the group has made a territorial call. Note that $L_{i}$ and $C_{i}$ need not be integer multiples of $l$, so the otter groups will not always end the time-step on an integer multiple of $l$ (i.e. this is a continuous-space model, not a lattice model).

Calls are assumed to extend the territory for a fixed time, $t_{C}$ (which we assume, for convenience, to be an integer multiple of $\tau$ ). Thus, for a time $t_{C}$ after group 1 makes a territorial call, it will forage within the interval $\left[0, C_{1}\right]$ and group 2 will forage within the interval $\left(C_{1}, L\right]$, unless group
Fig. 2 Behavioural states of each otter group and triggers for state changes. Each group $i \in\{1,2\}$ can be in one of three states at any time: Calling, Foraging, or Scenting. Arrows between each pair of states are labelled to show what triggers the change of state 
2 makes a Call. Likewise, Group 2 will forage within the interval $\left[C_{2}, L\right]$ for a time $t_{C}$ after it has made a territorial call, and group 1 within $\left[0, C_{2}\right)$, unless group 1 makes a Call. In any other situation, group 1 forages within the interval $\left[0, L_{1}\right]$ and group 2 within $\left[L_{2}, L\right]$. A group will not make a repeated call within a time of $t_{C}$ since its last Call.

Each group will also have to maintain its latrine by switching from Foraging mode to Scenting mode occasionally. As such, we assume that if a group has not been in Scenting mode for a time $t_{L}\left(N_{i}\right)$ then it switches to Scenting (again we assume, for convenience, that $t_{L}\left(N_{i}\right)$ is an integer multiple of $\tau)$. The function $t_{L}\left(N_{i}\right)$ is a monotonic increasing function of the group size, $N_{i}$, to model the fact that larger groups are able to leave longerlasting scent marks (Kean et al. 2011).

The switch from either Foraging or Scenting to Calling occurs only when the otter group has a need to extend its territory to gain access to extra resources. To model this idea, we use the notion of a group's Energy, denoted $E_{i}(t)$ for group $i$ at time $t$. When $E_{i}(t) \leq 0$, this means that the group can no longer survive in its territory, so members either leave or die-off (at which point in time the simulation ends). We also let $E_{\max }$ be the maximum energy that an individual otter can have. Then the energy of a group changes over time via the following function

$E_{i}(t+\tau)=\left\{\begin{array}{l}\min \left\{N_{i} E_{\max }, E_{i}(t)+N_{i}\left(a l \bar{R}_{i}(t)-E_{\tau}-\mu E_{\max } I[t, t+\tau]\right)\right\}, \text { when Foraging, } \\ \min \left\{N_{i} E_{\max }, E_{i}(t)+N_{i}\left(\gamma a l \bar{R}_{i}(t)-E_{\tau}-\mu E_{\max } I[t, t+\tau]\right)\right\}, \text { otherwise. }\end{array}\right.$

Here, $E_{\tau}$ is the energy used for an individual to move and live for a time-step of length $\tau$ (per force this is a linear function of $\tau$ that goes to zero as $\tau$ goes to zero), $\bar{R}_{i}(t)$ is the mean amount of resources (i.e. food) available in the territory per unit length, $\gamma \in[0,1]$ is the proportion of resources up-taken when Scenting or Calling compared to when Foraging, $a \in[0,1]$ is the conversion of available resources to energy by each individual, $\mu$ is the mean proportion of the maximum energy lost per individual if the group interacts with another group, $I[t, t+\tau]=1$ if both groups are in the same spatial location at the same time in the interval $(t, t+\tau]$ (i.e. they 'interact'), and $I[t, t+\tau]=0$ otherwise. Interactions between otter groups are typically aggressive and often lead to injuries (Groenendijk et al. 2014; Leuchtenberger et al. 2015; Rosas and De Mattos 2003), so we model this as a significant loss of energy to the group. Note that these can only happen at the points in time when one group is going beyond its territory border-i.e. when it is on a Calling foray.

We assume that $\bar{R}_{i}(t)$ is an increasing function of territory size, denoted $M_{i}(t)$, so that the larger the territory, the more food is available per unit length. We choose the following functional form for $\bar{R}_{i}(t)$, which is justified in Supplementary Appendix A.

$\bar{R}_{i}(t)=\max \left\{0, K\left[1-\frac{d\left(N_{i}\right)}{r} \frac{l}{M_{i}(t)-l}\right]\right\}$.

Here, $K$ is the carrying capacity of the river per unit length, $d\left(N_{i}\right)$ is the proportion of resourced depleted by $N_{i}$ animals in time $\tau$ (see Supplementary Appendix A), which is an increasing function of $N_{i}$, and $r$ is the resource renewal factor (see Supplementary Appendix A). The intuitive idea is that, in a self-renewing resource landscape, the larger the area that a group of animals has to live in, the more time the resources spend being renewed rather than depleting, and so the higher the quantity of the resources (on average) at any point in space. Supplementary Appendix A demonstrates mathematically that this intuition makes sense.

Group $i$ will switch from Foraging to Calling if $E_{i}(t) \leq$ $\lambda N_{i} E_{\max }$, for some fixed value $\lambda \in[0,1]$. Table 1 gives a list of all the parameters used in the model. Figure 2 summarises the movement processes in the various behavioural states, together with the situations that cause switches to occur between these states.

There are some implicit time-scales in this model worth emphasising. First, we assume the model is only valid across a maximum time of a single season, since we are not explicitly modelling seasonal fluctuations in resource availability. Furthermore, the model is only valid as long as the energies, $E_{i}(t)$, of both groups are above zero, since once either goes below zero, it leaves the system. In simulations (described later), we stop the system if the energy of either group goes below zero, since this indicates that the group has left the system. In summary, the model is valid across whichever is the shorter time-period: a single season (typically around 3 months) or the time until one group's energy goes below zero.

\section{Model analysis}

We split our analysis into three sections. We begin by assuming that otters do not use the Calling tactic to enlarge their territory (No Calling). In "Calling and scenting", we relax this assumption for one of the groups, to assess 
Table 1 Glossary of symbols used in the model

\begin{tabular}{|c|c|}
\hline Symbol & Definition \\
\hline$L$ & Length of spatial domain \\
\hline$\tau$ & Time-step duration (persistence time of otter movement) \\
\hline$l$ & Distance moved during a time-step of duration $\tau$ \\
\hline$L_{1}$ & Right-hand side of the latrine area for group 1 \\
\hline$L_{2}$ & Left-hand side of the latrine area for group 2 \\
\hline$C_{i}$ & Calling site of group $i(i=1,2)$ \\
\hline$N_{i}$ & Number of otters in group $i$ \\
\hline$t_{L}(N)$ & Time between trips to the latrine for a group of size $N$ \\
\hline$t_{C}$ & Time for which a territory call remains active \\
\hline$E_{i}(t)$ & Energy of group $i$ at time $t$ \\
\hline$E_{\max }$ & Maximum energy for an individual otter \\
\hline$E_{\tau}$ & Metabolic cost of an individual moving for a duration $\tau$ \\
\hline $\bar{R}_{i}(t)$ & Resources available to group $i$ per unit length at time $t$ \\
\hline$\gamma$ & $\begin{array}{l}\text { Proportion of resources up-taken when Scenting or Calling compared to when } \\
\text { Foraging }\end{array}$ \\
\hline$a$ & Proportion of available resources converted to energy by each individual \\
\hline$\mu$ & Proportion of $E_{\max }$ lost per individual in an inter-group encounter \\
\hline$M_{i}(t)$ & Length of territory group $i$ at time $t$ \\
\hline$d(N)$ & Resource depletion factor for a group of size $N$ \\
\hline$K$ & Carrying capacity of the environment per unit length \\
\hline$r$ & Resource renewal factor \\
\hline$\lambda$ & Proportion of maximum energy below which a group will switch to Calling \\
\hline$\Lambda_{i}$ & Dimensionless parameter $\Lambda_{i}=L_{i} / l$ \\
\hline$\Lambda$ & Dimensionless parameter $\Lambda=L / l$ \\
\hline$\Gamma_{i}$ & Dimensionless parameter $\Gamma_{i}=C_{i} / l$ \\
\hline$\alpha$ & Dimensionless parameter $\alpha=a K l$ \\
\hline$D(N)$ & Dimensionless parameter $D(N)=d(N) / r$ \\
\hline$T_{L}(N)$ & Dimensionless parameter $T_{L}(N)=t_{L}(N) / \tau$ \\
\hline$T_{C}$ & Dimensionless parameter $T_{C}=t_{C} / \tau$ \\
\hline$\delta$ & If $D(N) / N$ is constant then $\delta=D(N) / N$ \\
\hline$\epsilon$ & If $T_{L}(N) / N$ is constant then $\epsilon=T_{L}(N) / N$ \\
\hline
\end{tabular}

the benefit of a Calling strategy. "Calling competition" examines the case where both groups use both Calling and Scenting.

\section{No Calling}

In this section, we examine the conditions under which a territory will be viable without making Calling trips. We assume both territories are of equal length, so that $L_{1}=$ $L-L_{2}$, and that they are adjacent but non-overlapping ( $\left.L_{1}=L_{2}\right)$ so encounters between otter groups never occur. Due to the lack of such encounters, it suffices to focus on group 1. To model the lack of Calling behaviour, we set $\lambda=0$.
In this situation, it is possible to calculate the energy gain per time-step per individual, $E_{G}$, exactly. By introducing certain dimensionless composite parameters (see Table 1), we arrive at the following expression (derived in Supplementary Appendix B)

$$
E_{G}=\frac{\alpha}{2 T_{L}\left(N_{1}\right)+\Lambda_{1}}\left(1-\frac{D\left(N_{1}\right)}{\Lambda_{1}-1}\right)\left[\gamma \Lambda_{1}+2 T_{L}\left(N_{1}\right)\right]-E_{\tau},
$$

which holds as long as the energy level is sufficiently lower than $E_{\max }$. If $E_{G} \geq 0$ then the otter group is able to survive in perpetuity, without Calling. 
It is instructive to examine the edge cases, where $\gamma=1$ and $\gamma=0$. When $\gamma=1$, otters are assumed to intake as much energy from food whilst Scenting as they do when Foraging. Here

$E_{G}(\gamma=1)=\alpha\left(1-\frac{D\left(N_{1}\right)}{\Lambda_{1}-1}\right)-E_{\tau}$,

so that, since $D\left(N_{1}\right)$ is increasing, there may be a maximum group size $N_{1}$ for which $E_{G} \geq 0$, but no minimum. This maximum occurs because there is an upper limit to how crowded a particular territory can get (i.e. how many individuals can be sustained by the available resources).

For the case $\gamma=0$, so that otters do not gain any energy from food whilst Scenting, we have

$E_{G}(\gamma=0)=\frac{2 \alpha T_{L}\left(N_{1}\right)}{2 T_{L}\left(N_{1}\right)+\Lambda_{1}}\left(1-\frac{D\left(N_{1}\right)}{\Lambda_{1}-1}\right)-E_{\tau}$.

In this case, because $D\left(N_{1}\right)$ and $T_{L}\left(N_{1}\right)$ are both increasing, it is possible to have both a minimum and a maximum possible group size for which $E_{G} \geq 0$. This minimum emerges from a spatially explicit accounting of the costs and benefits of territory maintenance and exploitation. We call the set of values of $N_{1}$ for which $E_{G} \geq 0$ the Feasible Region for group sizes (Fig. 3).

To explore this idea, we examine the case where $T_{L}\left(N_{1}\right)=\epsilon N_{1}$ and $D\left(N_{1}\right)=\delta N_{1}$, so that these are both linear functions. Here, we see that increasing $L_{1}$ has the effect of increasing both the maximum and minimum of the Feasible Region, so that larger territories both require more individuals to maintain them and can sustain more individuals (Fig. 3a,b). Increasing $\alpha$, the uptake of energy by the otters from the environment, widens the Feasible Region (Fig. 3a,c). The parameter $\delta$ measures the rate at which each individual depletes resources. The larger $\delta$ is, the narrower the Feasible Region (Fig. 3a,d). In particular, if $\delta$ is too high, then the Feasible Region may contain no integer values, meaning that the territory is not viable (e.g. this happens for $\left.L_{1}=5, \alpha=40, \delta=0.8, \epsilon=2, E_{\tau}=15\right)$.

Increasing $\epsilon$, the scent longevity per individual in the group, also widens the Feasible Region (Fig. 3a,e). Conversely, a decrease in $\epsilon$ narrows the region, eventually leading to the case where the region contains no integer values. Finally, increasing $E_{\tau}$, the energetic cost of living and moving, has the effect of moving the curve down (Fig. 3a,f). If $E_{\tau}$ is too high then, as with small $\epsilon$ or large $\delta$, the Feasible Region will vanish.

\section{Calling and scenting}

For group sizes outside the Feasible Region (Fig. 3a), if otters take a pure-Scenting strategy, their energy will tend to decay over time. If this decay is sufficiently slow, otters may be able to survive a single 'lean' season, where the available forage is low, and then regain energy during a 'fat' season, if the forage then is sufficient for $E_{G}$ to be greater than zero (see Eq. 3). However, it may be the case that the decay in energy is too fast to survive a 'lean' season by taking a pureScenting strategy. In this section, we explore whether it is possible for otters to use Calling as a strategy to extend the time they are able to survive with fewer resources. Recall that the model is valid until either the season ends or one of the groups can no longer survive in the area, whichever is sooner. Therefore the underlying question (albeit addressed implicitly rather than explicitly) is whether Calling can extend the survival time of a group sufficiently so that a 'lean' season ends before the group is forced out of the area due to energy loss.

To analyse this situation, we begin by performing some approximate analytic calculations to determine the conditions under which Calling may enable the otter group to survive longer. We then back these up with simulations. To simplify our analysis, we assume that group 2 has a viable territory without needing to temporarily extend their territory with vocalisations. This means that, at any point in time, we can have two possible territorial configurations: (i) group 1's territory is $\left[0, C_{1}\right)$ and group 2 's is $\left(C_{1}, L\right]$, which occurs for a time of $t_{C}$ after a Call is made, or (ii) group 1's territory is $\left[0, L_{1}\right)$ and group 2's is $\left(L_{1}, L\right]=$ $\left(L_{2}, L\right]$, which is the situation at any other time (recall the assumption that $L_{1}=L_{2}$ ).

By making certain mean-field assumptions, it is possible to derive an inequality that approximates the region where there is some benefit to the group for employing a Calling strategy (see Supplementary Appendix C). For $\gamma=0$, this inequality is as follows

$$
\begin{gathered}
T_{C}\left[\alpha\left(1-\frac{D\left(N_{1}\right)}{\Gamma_{1}-1}\right)-\frac{2 \alpha T_{L}\left(N_{1}\right)}{2 T_{L}\left(N_{1}\right)+\Lambda_{1}}\left(1-\frac{D\left(N_{1}\right)}{\Lambda_{1}-1}\right)\right]> \\
\mu E_{\max } \frac{\Gamma_{1}-\Lambda_{2}}{\Lambda-\Lambda_{2}}+\frac{2 \Gamma_{1}-\Lambda_{1}}{2}\left(1-\frac{D\left(N_{1}\right)}{\Lambda_{1}-1}\right) \frac{2 \alpha T_{L}\left(N_{1}\right)}{2 T_{L}\left(N_{1}\right)+\Lambda_{1}} .
\end{gathered}
$$

By examining numerically the minimum value of $T_{C}$ at which this inequality holds (i.e. the value of $T_{C}$ for which this becomes an equality), we can observe how the various parameters affect the possibility of a Calling strategy being beneficial (Fig. 4a, solid lines). In general, the inequality in Eq. 6 predicts that as the calling point $C_{1}$ moves deeper into the foreign territory, so the minimum required longevity of the calling cue $\left(T_{C}\right)$ decreases. (Note, more precisely, that $T_{C}$ is the call longevity divided by movement persistence time, $\tau$ : see Table 1 ). However, because the inequality in Eq. 6 relies on various mean-field assumptions, it is necessary to perform stochastic simulations to verify its validity. 
Fig. 3 Regions where a no-Calling strategy is sufficient for survival. For each set of parameter values $L_{1}, \alpha, \delta, \epsilon, E_{\tau}$, there is a finite set of possible group sizes that can survive within a territory without the need for using a Calling strategy. This is labelled in each panel as the Feasible Region. Panel a plots this for a fixed set of parameters. Each other panel shows the effect of changing a single parameter as follows: b $L_{1}, \mathbf{c} \alpha, \mathbf{d} \delta, \mathbf{e} \epsilon, \mathbf{f} E_{\tau}$
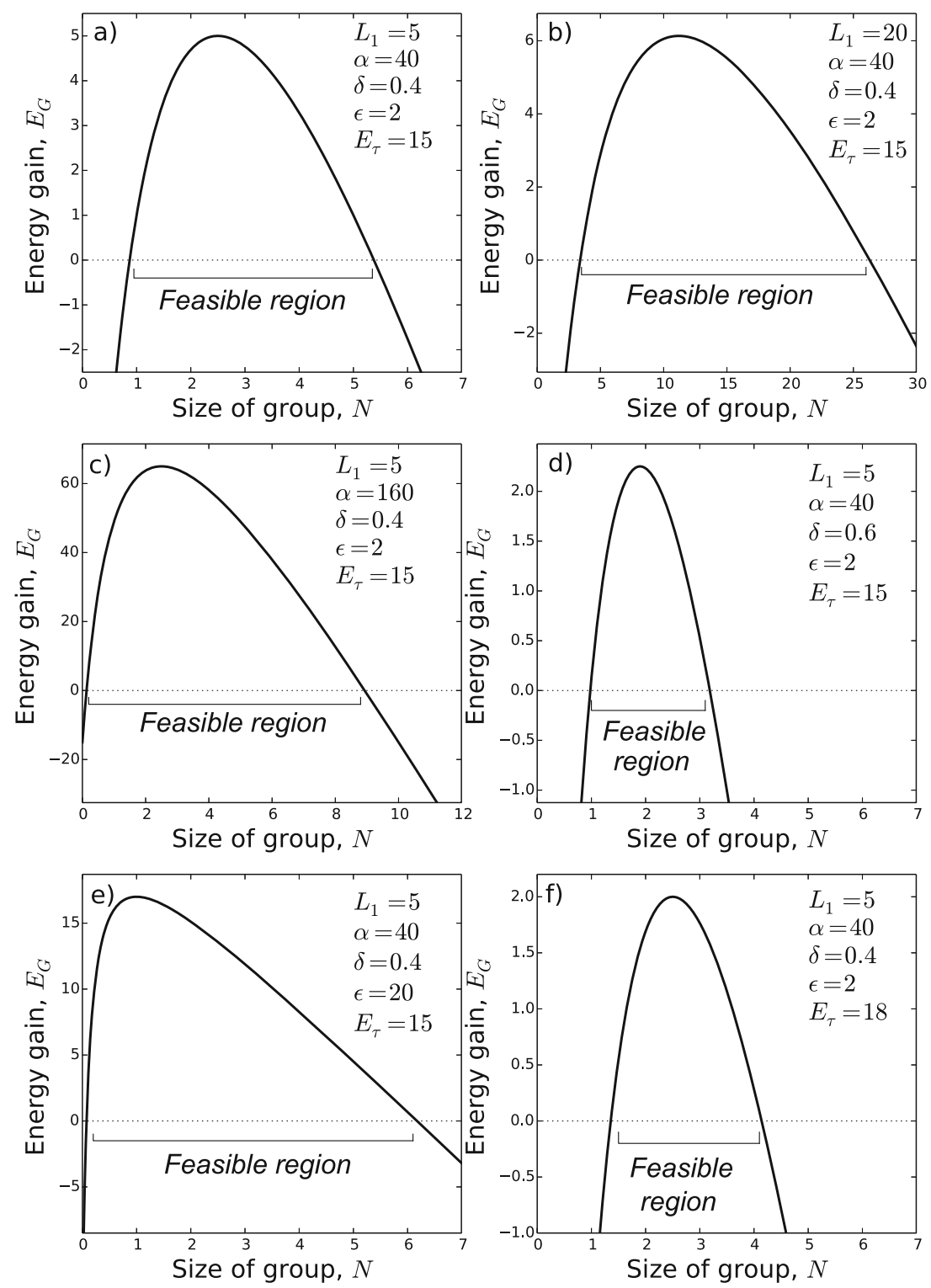

Simulations require that we pick particular parameter values to test. The most interesting cases occur when a pureScenting strategy is insufficient for group survival within a territory. In such cases, we explore the conditions under which a Calling strategy will be preferable and how much benefit an otter group can obtain from such a strategy. For this exploration, we fix parameter values $\delta=0.4, \epsilon=$ $20, E_{\tau}=15, \alpha=40, L_{1}=5, L_{2}=5, L=10, E_{\max }=$ $500, \mu=0$. We then vary $\lambda, N, C_{1}$, and $T_{C}$, and measure the time, $T_{\mathrm{fin}}\left(\lambda, C_{1}, T_{C}\right)$, until the energy of group 1 reaches 0 .

The value $T_{\text {fin }}\left(\lambda, C_{1}, T_{C}\right)$ can be thought of as the mean survival time of a group. This follows the model setup (The model) that an energy level of zero means that the individuals in the group leave the territory, either by dying or moving away from the area. If there is a $\lambda=$ $\lambda_{*}>0$ such that, when averaged over many simulations, $T_{\text {fin }}\left(\lambda_{*}, C_{1}, T_{C}\right)>T_{\text {fin }}\left(0, C_{1}, T_{C}\right)$ then we conclude that a Calling strategy is beneficial compared to a pure-Scenting strategy. We used 1,000 simulation runs, for each set of parameter values, to calculate these averages.

As anticipated by Inequality (6), for each value of $C_{1}$, there turns out to be a threshold value of $T_{C}$ above which Calling is beneficial. This threshold $T_{C}$ decreases as $C_{1}$ is increased (Fig. 4a), which is also anticipated by Inequality (6). Yet, the precise rate at which the threshold $T_{C}$ decreases is rather different in the stochastic simulations than in the analytic approximation from Inequality (6).

This is not surprising, however, since one of the stochastic aspects of the model is that an interaction confers a large loss in energy. Furthermore, once the energy reaches zero the simulation is stopped. Therefore, if a few 
interactions occur in a row, the simulation ends much earlier than in the case where otters, by chance, do not interact with the other group for several Calling trips in a row.

In the cases where Calling improves the ability for the otters to survive, the extent of this benefit can be quite pronounced, with certain parameter values showing an increase in the expected survival time of the group of several orders of magnitude (Fig. 4b). The greater the call longevity, the greater the benefit incurred from using a Calling strategy. Indeed, the sharp increase in the curve $C_{1}=7$ that occurs around $T_{C}=60$ indicates that increasing $T_{C}$ sufficiently may allow otters to survive indefinitely when $C_{1}$ is high. Therefore, as long as neighbouring otter groups are sufficiently intimidated by calls to stay away for a long time, this strategy could be sufficient to ensure otters survive a 'lean' season where otherwise they might have not.

If we incorporate a penalty for inter-group interactions, by setting $\mu=0.1$, then we still see an increase in expected survival time, sometimes of several orders of magnitude (Fig. 4c). (Note that even if group 2 can survive within a subordinated territory, $\left(C_{1}, L\right]$, it may still fight a territorial intrusion as territory size is a display of dominance, so there may yet be a penalty for inter-group interactions). However, the increase in survival time is less pronounced than without such a penalty (compare Panels b and c of Fig. 4). Notice that when $C_{1}$ is higher, there is a greater decrease in survival time as $\mu$ is increased. This is because there is a much higher chance of interactions when $C_{1}$ is higher. On the other hand, when $C_{1}$ is close to $L_{1}=5$, the overlap of territories is smaller so interactions are less frequent. Hence the penalty incurred by setting $\mu$ higher is only minimal.

We also investigated the benefits of a Calling strategy when the resources are not uniformly distributed across each territory. For this, we examined two cases: one where resources tend to be concentrated towards the centre of each territory and another where they are concentrated towards the border between territories (see Supplementary Appendix D for details). In the former case, the benefits of a Calling strategy were relatively similar to the case of uniform resources. However, in the latter case the benefits of a Calling strategy were much more pronounced even than in the case of uniform resources (Supplementary Fig. S1). This suggests that we may be more likely to observe Calling strategies in situations where resources are concentrated towards territory borders.

\section{Calling competition}

In "Calling and scenting", we assumed that group 2 can always survive with a pure-Scenting strategy and so never makes Calls. In this section, we examine the case where group 2 is unable to survive if group 1 makes Calls. Therefore, group 2 is forced to respond in kind by making Calls to extend its territory. For this, we fix parameter values $\delta=0.4, \epsilon=20, E_{\tau}=15, \alpha=40, L_{1}=5, L_{2}=$ $5, L=10, E_{\max }=500, \mu=0, N_{1}=9, N_{2}=4$ (see
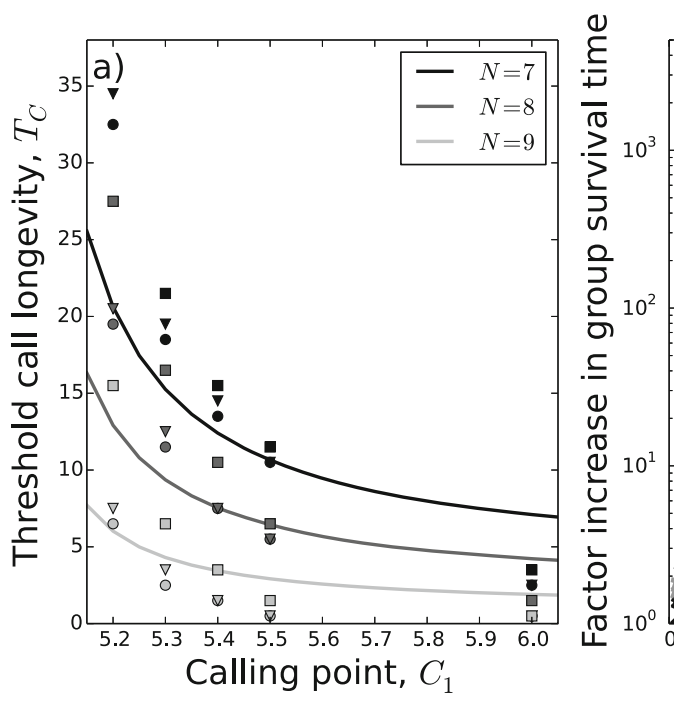

Fig. 4 The benefits of employing a Calling strategy. As long as the call longevity, $T_{C}$, is sufficiently large, a Calling strategy can enable otter groups to survive in conditions where a pure-Scenting strategy would not. In panel a, the solid lines show the analytic prediction of the minimum $T_{C}$ required for a Calling strategy to be beneficial (the minimum $T_{C}$ such that Inequality 6 holds). The circles give the minimum $T_{C}$ for which the group's survival time is greater with Calling than without (from simulation output). Triangles (resp. squares) show the minimum $T_{C}$ for which the group's survival time is more than $1 \%$ (resp. $5 \%$ )

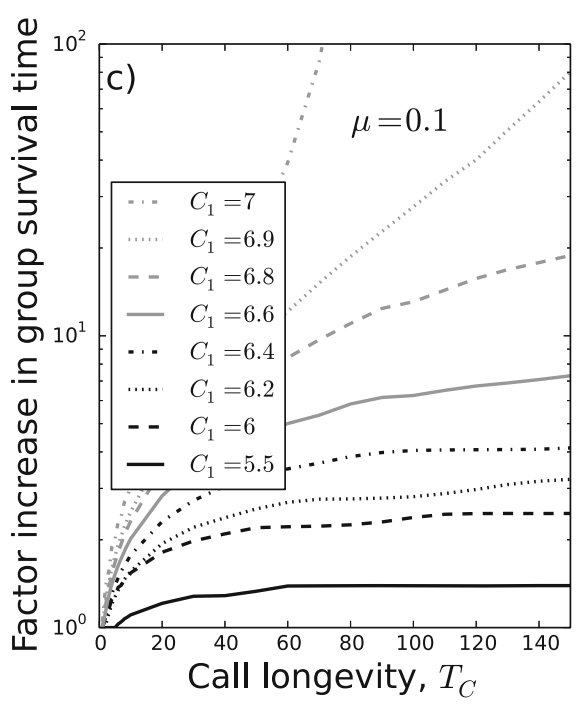

greater with Calling than without (from simulation output). Panel $\mathbf{b}$ shows the factor, $T_{\text {fin }}\left(\lambda_{*}, C_{1}, T_{C}\right) / T_{\text {fin }}\left(0, C_{1}, T_{C}\right)$ by which a Calling strategy is able to increase the survival time of a group, for different values of $T_{C}$ and $C_{1}$, when $N=9$ and $\mu=0$. Here, $\lambda_{*}$ is the value of $\lambda$ that maximises $T_{\text {fin }}\left(\lambda, C_{1}, T_{C}\right)$. If otters are able to push back their competitors a significant way with Calling, then they can increase their survival chances by several orders of magnitude. Panel $\mathbf{c}$ is the same as panel $\mathbf{b}$ except $\mu=0.1$, so there is a penalty for inter-group interactions (note the substantial change in the y-axis scale) 
Supplementary Appendix E for an analysis of the sensitivity of our results to small changes in these values). Note in particular that $N_{1} \neq N_{2}$ here. The value of $C_{2}$ is set so that the distance between the Calling point and the edge of the latrine area of each group is the same, i.e. $C_{1}-L_{1}=L_{2}-C_{2}$ (recall again that we are assuming $L_{1}=L_{2}=L / 2$ ). Simulations are run until the energy of either one of the groups has reached zero. The group that remains is said to have 'won'. The group that wins is subsequently able to survive in perpetuity by making use of the whole domain $[0, L]$.

In this competitive situation, it can still be beneficial to employ a Calling strategy (Fig. 5). However, unlike in "Calling and scenting", the benefit to both groups of extending the territory does not increase linearly with $C_{1}$. Instead, there is an intermediate length, $C_{1}=6.5$, that is optimal for increasing co-existence time (the time until one or other group wins, Fig. 5, left-hand panel). Should groups attempt to extend their territory beyond this length, the expected co-existence time lowers. Perhaps part of the reason for this is that when $C_{1} \lesssim 6.5$, group 2 almost always wins (Fig. 5, right-hand panel). However, for $C_{1} \gtrsim 6.5$, there is often a chance of group 1 winning. Therefore the decline in co-existence time for $C_{1} \gtrsim 6.5$ may be due to the energy of group 2 reaching zero faster as $C_{1}$ increases. This increased tendency for group 1 to win as $C_{1}$ is increased may be due to the fact that group 1 will always be the first group to extend its territory (since, until group 1 extends, group 2 has no need to Call). If the extension is made sufficiently far, group 1 gains the upper hand over group 2 from the outset, allowing it to win. However, if the extension is too short (i.e. $C_{1} \lesssim 6.5$ ), then no matter how long group 1 is able to maintain the extended territory (i.e. no matter the size of $T_{C}$ ), group 1 almost always ends up losing, which explains the monotonic appearance of the two left-hand insets in the left-hand panel of Fig. 5.

Similarly, in the situation described in "Calling and scenting", the greatest increase in survival time is conferred by having $\lambda=1$, so that group 1 is attempting to keep its territory permanently extended. However, the central panel of Fig. 5 shows that this is no longer always the case when adjacent groups are both making territorial Calls. On the contrary, there is often some intermediate value of $\lambda$, between 0 and 1 , which confers the maximal increase in co-existence time. These intermediate values occur more frequently for higher $T_{C}$ and $C_{1}$. In these cases, there is also more chance of group 1 winning, whereas with lower $T_{C}$ and $C_{1}$, group 2 almost always wins (Fig. 5, right-hand panel).

We also investigated a situation of symmetric competition, where all the parameters are as above but $N_{2}$ is the same size as $N_{1}$. Here, a Calling strategy was almost never beneficial for improving the co-existence time of the groups, and where there was some improvement, it was only minimal (see Supplementary Fig. S2). This demonstrates that Calling only works as a strategy if there is sufficient disparity between the groups, either in terms of group size or in terms of core territory size.

\section{Discussion}

We have constructed a model of moving territorial agents that demarcate their territory both by long-lasting scent depositions in fixed latrines and shorter-lasting vocalisations (which we term 'Calls') beyond their latrines. Our model of each 'agent' is based on a single territorial group of giant otters, but the model structure is quite general and should be applicable to many other species. The Calling strategy is high-risk compared to Scenting, due to the possibility of aggressive encounters. Indeed, our model shows that Calling need not be employed when the area demarcated by latrines is sufficient for the energetic needs of the group. This corroborates field observations, where territories are contiguous and non-overlapping in times when resources are plentiful (Leuchtenberger et al. 2015).

Because giant otter groups require more energy when they are larger, there is a maximum size of group that can be sustained by a given territory when taking a pure-Scenting strategy. However, larger groups can leave scent deposits that last for a longer time than smaller groups (Kean et al. 2011). As such, a minimum group size also emerges from the model (Fig. 3). This leads to a Feasible Region of group sizes, outside of which giant otter groups cannot survive in their territory without using a Calling strategy to extend their territory temporarily.

When Scenting is insufficient for survival, Calling strategies that temporarily expand a territory can be remarkably effective, increasing the survival time of a group by (in theory) up to several orders of magnitude, even when there is a significant penalty for interactions (Fig. 4c). This explains why such a strategy is often observed, and confirms the observations of spatially implicit models regarding the effectiveness of intrusion strategies (Hinsch and Komdeur 2010). Indeed, our spatially explicit approach goes further by linking the effectiveness of intrusion to the spatial extent of the resulting territorial border fluctuations, and hence the extent to which the resulting utilisation distributions will overlap when measured over time (Potts et al. 2012). This supports field observations that show overlapping territories when there might be less resource available (Leuchtenberger et al. 2015).

Calling is not a single-fix solution, though. Due to the increased probability of aggressive interactions, as well as the likelihood that adjacent groups will respond by making Calling trips of their own, Calling can fail to increase 

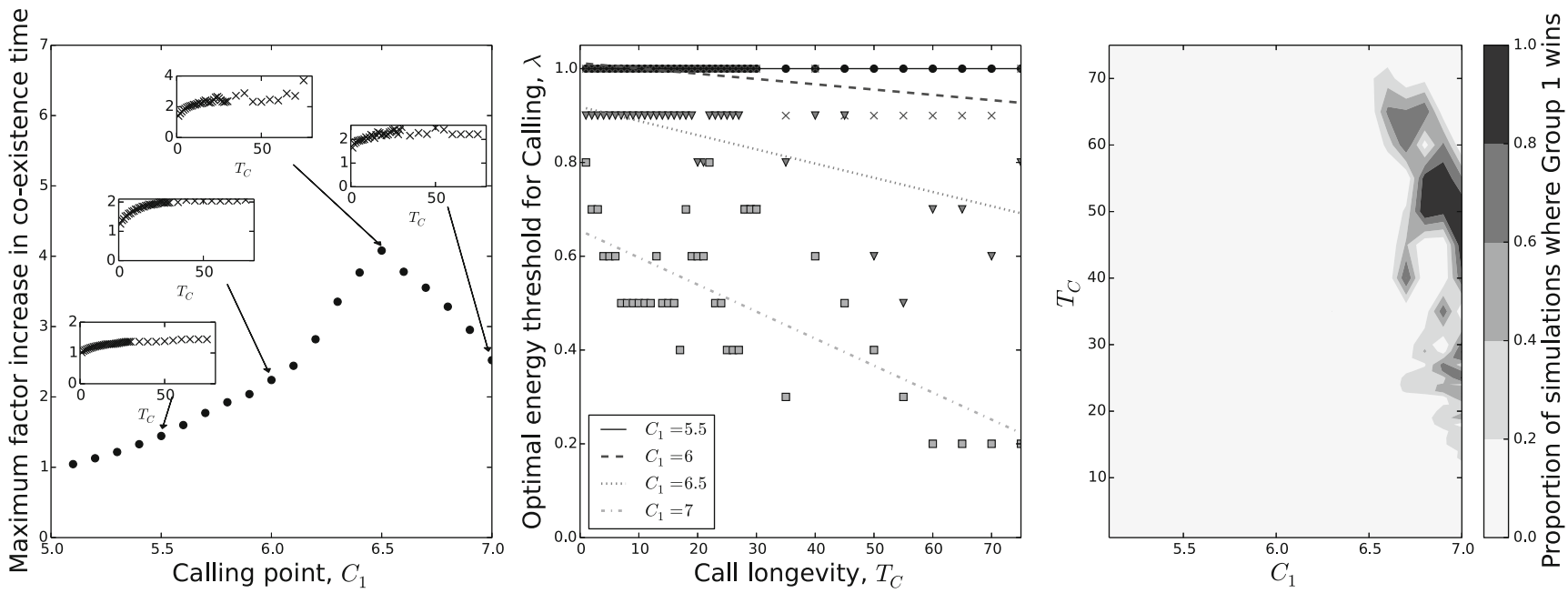

Fig. 5 Both groups employ a Calling strategy. Here, group 1 cannot survive without a Calling strategy, as long as group 2 is present. However, if group 1 extends its territory by Calling then group 2 will need to employ a Calling strategy in return. The left-hand panel shows the maximum factor by which a Calling strategy can increase the time during which both groups can co-exist, dependent on the position of the Calling point, $C_{1}$. At $C_{1}=6.5$, the increase in co-existence time is maximised. The central panel shows the values of $\lambda$ for which the

group longevity or even be detrimental to survival. In these situations, one group will eventually be forced to leave the area (through death or dispersal). The other group may then stand a better chance of survival by making use of the combined length of both the former territories. This latter group ultimately 'wins' the competition for resources.

In these competitive situations, the spatial nature of the modelling reveals a surprising non-monotonicity in the relationship between the location of the calling point and the ability for two competing groups to co-exist (Fig. 5). A small amount of territorial extension will mean that one group will die out quickly, typically the group that is unable to survive using a pure-Scenting strategy (group 1 in our example). A better strategy for group 1 appears to be to attempt a large territorial extension, which can result in it overpowering group 2 rapidly (right-hand panel, Fig. 5), thus also leading to a lower mean co-existence time. However, an intermediate distance means that group 1 can extend its survival time for a comparatively long time, without forcing group 2 out of the region.

Although it is clear that this intermediate distance is better for the population collectively, what constitutes the most beneficial strategy for an individual group (which is what we are more likely to see in reality) remains an open question. Answering this would require a game-theoretic analysis, whereby each group varies the distance at which it seeks to extend its territories, in response to the other's Calling forays. Whilst beyond the scope of the present paper, our model and results provide a theoretical basis for such analysis. We therefore hope to make this the subject co-existence time is maximised, for various values of $T_{C}$ and $C_{1}$. The simulation output is given by dots $\left(C_{1}=5.5\right)$, crosses $\left(C_{1}=6\right)$, triangles $\left(C_{1}=6.5\right)$ and squares $\left(C_{1}=7\right)$. Respective best-fit lines are also shown (see figure legend). The right-hand panel shows the proportion of simulations where group 1 not only increases its survival time, but actually wins by out-competing and eliminating group 2 , as a function of $T_{C}$ and $C_{1}$

of future work. Such game-theoretic explorations could also incorporate factors that have not been explicitly modelled here, such as a link between the group size and both the Call longevity $\left(T_{C}\right)$ and the length of the foray (derived from $C_{1}$ and $C_{2}$ ). In general, many extensions to the model are possible (e.g. into oxbow-lakes or other water-bodies that have different geometries to a narrow river). The aim of the present manuscript is to provide a solid basis on which these can be built, one at a time, to understand the effect of each on the spatial population dynamics.

A variety of papers discuss territoriality from a gametheoretic perspective but do not explicitly model the movement of animals. A possible fruitful avenue of research would be to incorporate these into our movementand-interaction framework. For example, Pereira et al. (2002) examine the division of space that emerges from a negotiation between neighbours who may be asymmetric in defence abilities. Mesterton-Gibbons and Adams (2003) analyse territorial conflict when groups have imperfect information about their neighbours and resolve disputes by making use of fixed landmarks to demarcate territory borders. Adler and Gordon (2003) examine the territories that emerge from optimizing a model of resource availability, information, and neighbour behaviour, with specific application to ants. We have already mentioned the study of Hinsch and Komdeur (2010), which is closest to ours in the questions it asks, but its spatially implicit nature means that it is more limited in the range of answers it can provide. All of these may benefit from analysis within a movement framework, both to verify their predictions in 
a situation slightly closer to reality, and to examine any further results that can be obtained by modelling movement capabilities.

Furthermore, extensions may be made to our model to make the role of scenting in territorial defense more explicit. At the moment, the latrines are assumed to deter neighbouring groups in a pre-defined way, and it is also always assumed that these latrines will be regularly 'toppedup' so their ability to deter does not change over time. In reality, it may be the case that fresher scent cues in the latrines are a bigger deterrence to neighbouring groups, so may make them either less likely to perform an extraterritorial foray, or to perform a foray of a shorter distance. Modelling such a feature would explicitly tie in the values of $C_{i}$ to the time, $T_{L}(N)$, between latrine trips, so may be an interesting extension for future work.

As well as using Calling as a strategy for survival when food levels are low, our results also indicate that survival may be achieved by group fission/fusion events. For example, the Feasible Region in Fig. 3e suggests the group size, $N$, must be between 1 and 6 (inclusive). Hence, if a group of size 4 is adjacent to a group of size 8 then the latter group could not survive (given all the parameters from Fig. 3e). Yet, if two individuals leave from the larger group moved into the smaller then both groups would survive. Membership exchange is known to occur among giant otters (Groenendijk et al. 2015; Ribas et al. 2016), and adult helpers can leave their parental groups to become solitary transients or form groups of non-breeding transient otters, which have not obtained a territory yet (Groenendijk et al. 2014; Ribas et al. 2016). However, this situation is not the case for the parameters examined in "Calling competition", where $N_{1}=9, N_{2}=4$ and other parameters are as in Fig. 3e. In this case, swapping groups is not a beneficial strategy but Calling can be.

Territorial invasions are very tense moments for otters, and invaders spend a substantial fraction of their time in the neighbours' territory engaged in Calling and Scenting as they attempt to extend their territory. We have simplified this process somewhat to focus on the potential benefits of Calling during the territorial extension. Nevertheless, our approach captures the key element that intruders risk energy losses not just from hostile interactions with their neighbours but costs that arise from lost opportunities when they are in behavioural modes other than Foraging.

Calling strategies that facilitate temporary intrusions into another group's territory may be especially important when resources are concentrated towards territory borders (see Supplementary Appendix D). This result thus links conceptually with earlier results demonstrating that the interplay between territorial predators may actually cause prey to concentrate at the boundaries of territories (Lewis and Murray 1993). In such contexts, two modes of communication between predator groups (long-lived scent marks and transient calls) may work synergistically to enhance access to concentrated prey resources.

Territorial extensions have been studied previously in slightly different contexts. Tao et al. (2016) describe a model of territorial animals who have a specific central place around which they are localised, such as a den or nest site. In this model, rather than having clearly demarcated borders like giant otters, the space use decays exponentially from the central place. The rate of decay, and hence the home range size, is determined by a trade-off between defending the territory by staying closer to the central place and exploring more widely to extend the territory.

However, the lack of clearly defined borders in the model of Tao et al. (2016) makes it insufficient for the sort of analysis performed here. More generally, advectiondiffusion models rely on smooth functional forms so modelling sharp borders requires imposing them directly (Potts et al. 2012). Here, we make such an imposition, assuming that foraging otters are constrained to move within their borders. That said, the model of Tao et al. (2016) appears well-suited to populations where territorial borders do not have such a precise location, and particularly where the localisation of animals is largely driven by the existence of a central site of interest. The review by Potts and Lewis (2014) compares advection-diffusion approaches with models that explicitly incorporate sharp (but possibly moving) borders, in more generality.

In summary, our model gives plausible movement and interaction mechanisms that explain a variety of observed phenomena, including (a) the existence of overlapping and non-overlapping home ranges under different resource conditions, (b) the correlation between overlap, resource abundance, and extra-territorial vocalisations, (c) groups being of an intermediate size and (d) group fission/fusion events. It therefore serves as a test-bed for understanding when and why territorial animals will seek to extend their territory, as well as making predictions about the survival of populations should their food abundance change.

Acknowledgements We thank Nina Attias, Gustavo Santos, and Chris Fleming, who provided comments that helped frame the problem biologically. We thank various anonymous reviewers who provided helpful comments on the manuscript.

Funding information JRP receives support from the National Environmental Research Council (NERC) grant NE/R001669/1. WFF receives support from the US National Science Foundation (NSF) grant ABI 1458748. GM receives support from the Fundação de Apoio ao Desenvolvimento de Ensino, Ciência e Tecnologia do MS (FUNDECT) grant Pronex 006/2015

Open Access This article is distributed under the terms of the Creative Commons Attribution 4.0 International License (http:// creativecommons.org/licenses/by/4.0/), which permits unrestricted use, distribution, and reproduction in any medium, provided you give appropriate credit to the original author(s) and the source, provide a 
link to the Creative Commons license, and indicate if changes were made.

\section{References}

Adams ES (2001) Approaches to the study of territory size and shape. Ann Rev Ecol Syst 32(1):277-303

Adler FR, Gordon DM (2003) Optimization, conflict, and nonoverlapping foraging ranges in ants. Am Nat 162(5):529-543

Bartels PJ (1984) Extra-territorial movements of a perennially territorial damselfish, eupomacentrus dorsopunicans poey. Behaviour 91(4):312-321

Burt WH (1943) Territoriality and home range concepts as applied to mammals. J Mammal 24(3):346-352

Carter S, Rosas FC (1997) Biology and conservation of the giant otter pteronura brasiliensis. Mammal Rev 27(1):1-26

Craig P (1996) Intertidal territoriality and time-budget of the surgeonfish, acanthurus lineatus, in American Samoa. Env Biol Fish 46(1):27-36

Delahay R, Brown J, Mallinson P, Spyvee P, Handoll D, Rogers L, Cheeseman C (2000) The use of marked bait in studies of the territorial organization of the European badger (meles meles). Mammal Rev 30(2):73-87

Duplaix N (1980) Observations on the ecology and behavior of the giant river otter pteronura brasiliensis in Suriname. Revue d'Ecologie (La Terre et La Vie) 34:495-620

Evans ML, Stutchbury BJ, Woolfenden BE (2008) Off-territory forays and genetic mating system of the wood thrush (hylocichla mustelina). Auk 125(1):67-75

Evans P, Macdonald D, Cheeseman C (1989) Social structure of the eurasian badger (meles meles): genetic evidence. J Zool 218(4):587-595

Giuggioli L, Potts JR, Harris S (2011) Animal interactions and the emergence of territoriality. PLoS Comput Biol 7(3):e1002008

Giuggioli L, Potts JR, Harris S (2012) Predicting oscillatory dynamics in the movement of territorial animals. J Roy Soc Interface 9(72):1529-1543

Groenendijk J, Hajek F, Johnson PJ, Macdonald DW, Calvimontes J, Staib E, Schenck C (2014) Demography of the giant otter (pteronura brasiliensis) in manu national park, South-eastern Peru: implications for conservation. PloS One 9(8):e106202

Groenendijk J, Hajek F, Schenck C, Staib E, Johnson P, Macdonald D (2015) Effects of territory size on the reproductive success and social system of the giant otter, South-eastern Peru. J Zool 296(3):153-160

Hinsch M, Komdeur J (2010) Defence, intrusion and the evolutionary stability of territoriality. J Theor Biol 266(4):606-613

Kean EF, Müller CT, Chadwick EA (2011) Otter scent signals age, sex, and reproductive status. Chem Sens 36(6):555-564

Leuchtenberger C, Mourão G (2009) Scent-marking of giant otter in the southern Pantanal, Brazil. Ethology 115(3):210-216

Leuchtenberger C, Magnusson WE, Mourão G (2015) Territoriality of giant otter groups in an area with seasonal flooding. PloS One 10(5): $\mathrm{e} 0126073$

Lewis M, Moorcroft P (2006) Mechanistic home range analysis. Princeton University Press, Princeton

Lewis MA, Murray JD (1993) Modelling territoriality and wolf-deer interactions. Nature 366:738-740
Masoliver J, Lindenberg K, Weiss GH (1989) A continuoustime generalization of the persistent random walk. Physica A 157(2):891-898

Messier F (1985) Solitary living and extraterritorial movements of wolves in relation to social status and prey abundance. Can J Zool 63(2):239-245

Mesterton-Gibbons M, Adams ES (2003) Landmarks in territory partitioning: a strategically stable convention? Am Nat 161(5):685-697

Mitchell MS, Powell RA (2004) A mechanistic home range model for optimal use of spatially distributed resources. Ecol Model 177(12):209-232. https://doi.org/doi.org/10.1016/j.ecolmodel.2004. 01.015

Naguib M, Altenkamp R, Griessmann B (2001) Nightingales in space: song and extra-territorial forays of radio tagged song birds. J Ornithol 142(3):306-312

Patterson BR, Messier F (2001) Social organization and space use of coyotes in Eastern Canada relative to prey distribution and abundance. J Mammal 82(2):463-477

Pereira HM, Bergman A, Roughgarden J (2002) Socially stable territories: the negotiation of space by interacting foragers. Am Nat 161(1):143-152

Piper WH, Tischler KB, Klich M (2000) Territory acquisition in loons: the importance of take-over. Anim Behav 59(2):385-394

Potts JR, Lewis MA (2014) How do animal territories form and change? Lessons from 20 years of mechanistic modelling. Proc Roy Soc B 281(1784):20140231

Potts JR, Lewis MA (2016a) How memory of direct animal interactions can lead to territorial pattern formation. J Roy Soc Interface 13(118):20160059

Potts JR, Lewis MA (2016b) Territorial pattern formation in the absence of an attractive potential. J Math Biol 72(1-2):25-46

Potts JR, Harris S, Giuggioli L (2012) Territorial dynamics and stable home range formation for central place foragers. PLoS One 7(3): 34033

Ribas C, Mourão G (2004) Intraspecific agonism between giant otter groups. IUCN Otter Specialist Group Bull 21(2):89-93

Ribas C, Cunha HA, Damasceno G, Magnusson WE, Solé-Cava A, Mourão G (2016) More than meets the eye: kinship and social organization in giant otters (pteronura brasiliensis). Behav Ecol Sociobiol 70(1):61-72

Roper T, Shepherdson D, Davies J (1986) Scent marking with faeces and anal secretion in the European badger (meles meles): seasonal and spatial characteristics of latrine use in relation to territoriality. Behaviour 97(1):94-117

Rosas FCW, De Mattos GE (2003) Natural deaths of giant otters (pteronura brasiliensis) in balbina hydroelectric lake, Amazonas, Brazil. Otter Specialist Group Bulletin

Tao Y, Börger L, Hastings A (2016) Dynamic range size analysis of territorial animals: an optimality approach. Am Nat 188(4):460-474

Wilson RP, Griffiths IW, Legg PA, Friswell MI, Bidder OR, Halsey LG, Lambertucci SA, Shepard ELC (2013) Turn costs change the value of animal search paths. Ecol Lett 16(9):1145-1150. https://doi.org/10.1111/ele.12149

Winemiller KO, Jepsen DB (1998) Effects of seasonality and fish movement on tropical river food webs. J Fish Biol 53(sA):267-296

Wirtz P (1981) Territorial defence and territory take-over by satellite males in the waterbuck kobus ellipsiprymnus (bovidae). Behav Ecol Sociobiol 8(2):161-162

Zhou Y, Fagan WF (2017) A discrete-time model for population persistence in habitats with time-varying sizes. J Math Biol 75(3):1-56 